\title{
Composition dependence of bulk superconductivity in $\mathrm{YFe}_{2} \mathrm{Ge}_{2}$
}

\author{
Jiasheng Chen, ${ }^{1, *}$ Monika B. Gamża, ${ }^{2}$ Konstantin Semeniuk, ${ }^{1}$ and F. Malte Grosche ${ }^{1, \dagger}$ \\ ${ }^{1}$ Cavendish Laboratory, University of Cambridge, Cambridge CB3 OHE, United Kingdom \\ ${ }^{2}$ Jeremiah Horrocks Institute for Mathematics, Physics and Astronomy, University of Central Lancashire, Preston PRI 2HE, United Kingdom
}

(Received 1 October 2018; published 2 January 2019)

\begin{abstract}
In the layered iron-based superconductor $\mathrm{YFe}_{2} \mathrm{Ge}_{2}$, a high Sommerfeld ratio of $\sim 100 \mathrm{~mJ} / \mathrm{mol} \mathrm{K}$ and a $T^{3 / 2}$ temperature dependence of the electrical resistivity at low temperature $T$ indicate strong electronic correlations and point toward an unconventional pairing state. We have investigated the role of composition and annealing conditions in optimizing the growth of high-quality $\mathrm{YFe}_{2} \mathrm{Ge}_{2}$. Our findings confirm that bulk superconductivity is observed in samples with disorder scattering rates less than $2 k_{B} T_{c} / \hbar$. Fe deficiency on the Fe site is identified as the dominant source of disorder, which can be minimized by precipitating from a slightly iron-rich melt, followed by annealing.
\end{abstract}

DOI: 10.1103/PhysRevB.99.020501

The iron-based superconductor $\mathrm{YFe}_{2} \mathrm{Ge}_{2}$ [1] exhibits strong electronic correlations: its Sommerfeld ratio is enhanced by an order of magnitude over density functional theory (DFT) estimates [2-4], the normal state resistivity $\rho$ follows a non-Fermi-liquid temperature dependence, photoemission spectroscopy has revealed renormalized energy bands [5], and it displays enhanced magnetic fluctuations $[6,7]$. Further interest in this material derives from theoretical proposals for the superconducting state, which include $s_{ \pm}$ [3] or triplet pair wave functions [4] and from its striking similarities to some of the iron pnictide superconductors $[8,9]$. Moreover, several structurally and electronically related materials have recently been examined, some of which were found to superconduct at low temperatures [10-13], including a new iron-based superconductor, $\mathrm{LaFeSiH}$ [14].

Advanced experiments probing the low temperature state of $\mathrm{YFe}_{2} \mathrm{Ge}_{2}$ such as muon spin rotation, penetration depth, and quantum oscillation measurements have been held back by the lack of bulk superconducting, high-purity single crystals. Although a comprehensive growth study produced fluxgrown single crystals [15] with comparatively high residual resistivity ratios $\mathrm{RRR}=\rho(300 \mathrm{~K}) / \rho(2 \mathrm{~K}) \simeq 60$ and sharp resistive superconducting transitions, these did not display a superconducting heat capacity anomaly. Only a second generation of polycrystalline samples with even higher RRR provided thermodynamic evidence for bulk superconductivity in $\mathrm{YFe}_{2} \mathrm{Ge}_{2}$ [8].

In order to investigate the origin of this sample dependence, we have conducted a systematic study of the composition phase diagram of $\mathrm{YFe}_{2} \mathrm{Ge}_{2}$ and of the effect of annealing conditions on sample quality. Here, we present the key outcomes of this study, which may guide the preparation of high-quality, bulk superconducting single crystals and provide insights into the origin of superconductivity in $\mathrm{YFe}_{2} \mathrm{Ge}_{2}$, namely, (i) solidification from a slightly iron-rich melt followed by

\footnotetext{
*jc732@cam.ac.uk

${ }^{\dagger}$ fmg12@cam.ac.uk
}

quenching and annealing maximizes Fe occupancy on the Fe site and produces the samples with the longest electronic mean free path, (ii) $T_{c}$ is strongly reduced below its optimal value $T_{c 0}$ by disorder when the electronic scattering rate approaches $2 k_{B} T_{c 0} / \hbar$, and (iii) disorder and inhomogeneity induce Griffiths-phase signatures, suggesting close proximity of pristine $\mathrm{YFe}_{2} \mathrm{Ge}_{2}$ to magnetic instabilities.

Polycrystalline $\mathrm{Y}_{1+x}\left(\mathrm{Fe}_{1+y} \mathrm{Ge}_{1+z}\right)_{2}$ ingots (with $-0.1<$ $x, y, z<0.1)$ were grown in a radio-frequency induction furnace on a water-cooled copper boat under a high-purity Tigettered $\mathrm{Ar}$ atmosphere. To limit the precipitation of stable YGe alloys, Y (3N; Alfa Aesar) and Fe (4N, vacuum remelted; Alfa Aesar) were melted first and thoroughly mixed to form ingots of $\mathrm{YFe}_{2}$. Ge (6N; Alfa Aesar) and $\mathrm{Y}$ or Fe were then added and melted together with $\mathrm{YFe}_{2}$ to obtain the desired nominal composition. The mass losses due to evaporation were less than $0.3 \%$, and homogeneity was ensured by electromagnetic stirring and repeated flipping and remelting of the ingots. The ingots were quenched to the cooling water temperature within seconds and then heated up again to near $1250{ }^{\circ} \mathrm{C}$ for a first annealing step in the induction furnace, which was again followed by rapid quenching. Each of the resulting ingots was mechanically broken up into two halves, one of which was subsequently annealed at $800{ }^{\circ} \mathrm{C}$ in an evacuated quartz ampoule for 7 days ("annealed"), whereas the other half was investigated without prior annealing ("asgrown").

Heat capacity and electrical resistivity were measured with the helium-3 option of the Quantum Design Physical Properties Measurement System from 300 to $0.4 \mathrm{~K}$ using the pulserelaxation technique and a standard four-wire ac technique, respectively. The resistivity data were scaled at $300 \mathrm{~K}$ to the published value of $190 \mu \Omega \mathrm{cm}$ [2]. All the annealed samples and a selection of as-grown samples were measured to check for evidence of bulk superconductivity.

Powder X-ray diffraction (XRD) patterns were collected in the Bragg-Brentano geometry with $\mathrm{Cu} K \alpha$ radiation at $40 \mathrm{kV}$ and $40 \mathrm{~mA}$ on a Bruker D8 diffractometer equipped with a Lynxeye XE detector to reduce the effects of Fe fluorescence 


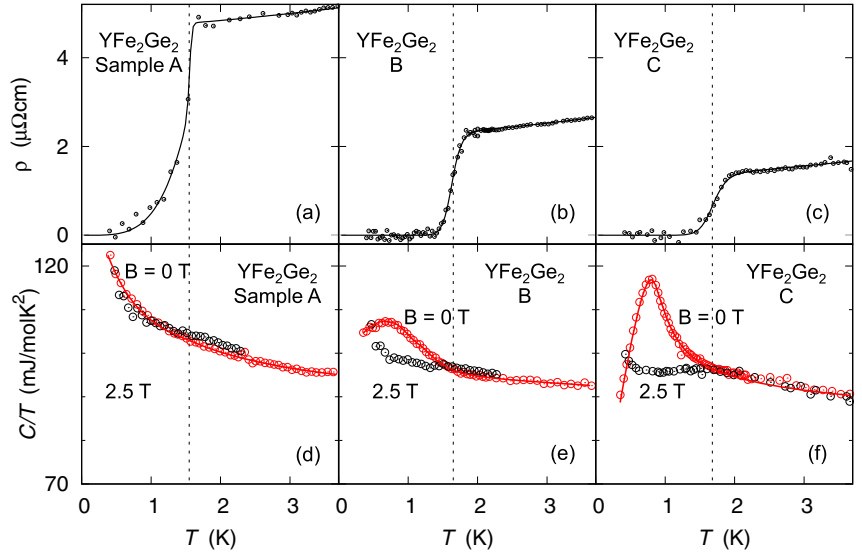

FIG. 1. Electrical resistivity $\rho$ [upper panels (a)-(c)] and Sommerfeld coefficient of the heat capacity $C / T$ [lower panels (d)(f)] for three typical samples of $\mathrm{YFe}_{2} \mathrm{Ge}_{2}$ with different residual resistivity $\rho_{0}$. Sample $A$ is as-grown but derives from the same ingot as a bulk superconducting annealed sample, for which data is presented in [8]. All three samples show resistive superconducting transitions, but a heat capacity anomaly indicating bulk superconductivity only appears in the purer, annealed samples B and C. When a superconducting heat capacity anomaly is absent (sample A), $C / T$ displays a slow increase on cooling even in magnetic fields sufficient to suppress $T_{c}$ fully, suggesting an underlying magnetic contribution.

and $K \beta$ radiation. Refinements of the powder patterns were carried out with FULLPROF. Lattice parameters were determined by referring to an internal Ge standard and using the Le Bail method. Multiple measurements were performed on selected batches of representative samples, in order to obtain estimates of the typical variation of lattice parameters. Energy dispersive spectroscopy (EDS) was measured with an Oxford $\mathrm{X}-$ Max detector in an FEI/Philips XL-30 ESEM at $30 \mathrm{kV}$ and analyzed with INCA software.

The key features of resistivity and heat capacity data are illustrated in Fig. 1. The as-grown (unannealed) sample A shows a resistive superconducting transition, but no superconducting anomaly in the heat capacity [Figs. 1(a) and 1(d)]. This sample derives from the same ingot as the annealed sample that showed the superconducting heat capacity anomaly in [8]. We find more generally that unannealed samples have low RRR values, and while some show a resistive transition all lack superconducting heat capacity anomalies [16]. By contrast, all of the annealed samples show resistive superconducting transitions with varying $T_{c}$, but not all exhibit signatures of bulk superconductivity in their specific heat (e.g., [1]). Distinct heat capacity anomalies, namely, broad jumps in $C(T) / T$ near $1 \mathrm{~K}$ with a peak at about $20 \%$ above the normal-state values, are observed in high-quality samples with RRR above 120 [Figs. 1(c), 1(f) and [8]]. Less prominent anomalies with peaks roughly $10 \%$ above the normal-state $C(T) / T$ can be found in samples with RRR ranging from 60 to 120, as illustrated in Figs. 1(b) and 1(e).

The influence of disorder scattering on superconductivity in $\mathrm{YFe}_{2} \mathrm{Ge}_{2}$ can be examined quantitatively using the large number of samples (annealed and as-grown) prepared from more than 20 ingots grown for this study. The dependence of the resistive $T_{c}$ on residual resistivity $\rho_{0}$ is summarized in

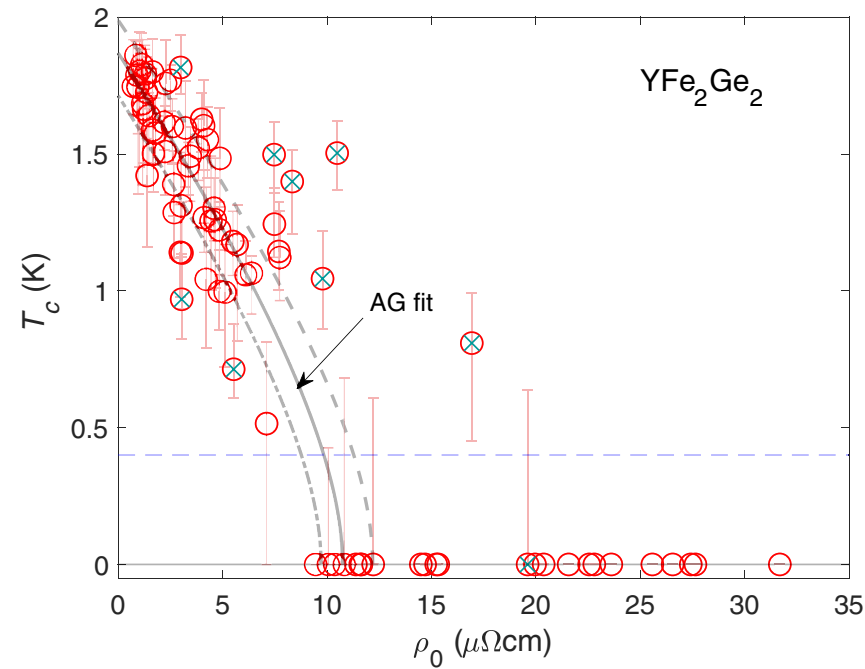

FIG. 2. Resistive transition temperature as a function of the residual resistivity $\rho_{0}$ for all $\mathrm{YFe}_{2} \mathrm{Ge}_{2}$ polycrystalline samples characterized in this study. No data are available below $0.4 \mathrm{~K}$ (indicated by the blue dotted line). Midpoints of the resistive transitions are shown as circles, with vertical error bars illustrating the transition widths (determined by an $80 \% / 20 \%$ criterion). Solid, dashed, and dash-dotted lines give least-square fits of the Abrikosov-Gor'kov expression (AG; see text) to the $50 \%, 80 \%$, and $20 \% T_{c}$ points, ignoring those outliers indicated by crosses.

Fig. 2, which illustrates that the data can be modeled by the implicit Abrikosov-Gor'kov expression [17]

$$
\ln \left(\frac{T_{c 0}}{T_{c}}\right)=\Psi\left(\frac{1}{2}+\frac{\alpha T_{c 0}}{2 \pi T_{c}}\right)-\Psi\left(\frac{1}{2}\right)
$$

where $T_{c}$ and $T_{c 0}$ are the actual transition temperature and the optimal transition temperature without impurity scattering, respectively, $\alpha \propto \rho_{0}$ measures the pair-breaking effect of impurity scattering, and $\Psi(z)$ is the digamma function. This approach has been found to describe the experimental data on cuprates $[18,19]$, the spin-triplet superconductor $\mathrm{Sr}_{2} \mathrm{RuO}_{4}$ $[20,21]$, and the heavy fermion superconductor $\mathrm{CeCoIn}_{5}[22]$. Impurity scattering is expected to suppress an unconventional pairing state, when the pair-breaking parameter $\alpha=\frac{1}{2} \frac{\hbar \tau^{-1}}{k_{B} T_{c 0}}$ approaches $1[18,23,24]$, where $\tau^{-1}$ is the quasiparticle scattering rate. Our data suggest an optimal $T_{c 0}$ of $1.87 \mathrm{~K}$ and show a clear trend for $T_{c}$ to diminish with increasing $\rho_{0}$ and superconductivity to be suppressed when $\rho_{0}>10.7 \mu \Omega \mathrm{cm}$.

The scattering rate can be estimated from $\rho_{0}$ using the Drude result $\tau^{-1}=\epsilon_{0} \Omega_{p}^{2} \rho_{0}$, where $\Omega_{p}$ is the renormalized plasma frequency, which is reduced with respect to the bare plasma frequency obtained from a DFT calculation, $\Omega_{p}^{(0)}$, by the ratio of effective mass $m^{*}$ over band mass $m_{0}$ : $\Omega_{p}^{2}=\left(\Omega_{p}^{0}\right)^{2} \frac{m_{0}}{m^{*}}$. Estimating $\left(\Omega_{p}^{(0)}\right)^{2}=\left[\left(\Omega_{x}^{(0)}\right)^{2}+\left(\Omega_{y}^{(0)}\right)^{2}+\right.$ $\left.\left(\Omega_{z}^{(0)}\right)^{2}\right] / 3 \simeq(3.43 \mathrm{eV} / \hbar)^{2}$ on the basis of DFT calculations [4], and taking the mass enhancement from the ratio of experimental Sommerfeld coefficient $\gamma_{\exp } \simeq 100 \mathrm{~mJ} / \mathrm{mol} \mathrm{K}^{2}$ over its DFT counterpart $\gamma_{0} \simeq 12.4 \mathrm{~mJ} / \mathrm{mol} \mathrm{K}^{2}$ [4] to be $\frac{m^{*}}{m_{0}} \simeq 8$, we find that $\hbar \tau^{-1}=\rho_{0} \epsilon_{0} \hbar \Omega_{p}^{2}=0.197 \mathrm{meV}\left(\rho_{0} / \mu \Omega \mathrm{cm}\right)$. For an optimum $T_{c 0} \simeq 1.87 \mathrm{~K}$, this gives $\alpha=0.62\left(\rho_{0} / \mu \Omega \mathrm{cm}\right)$, which would suggest that superconductivity should already be 

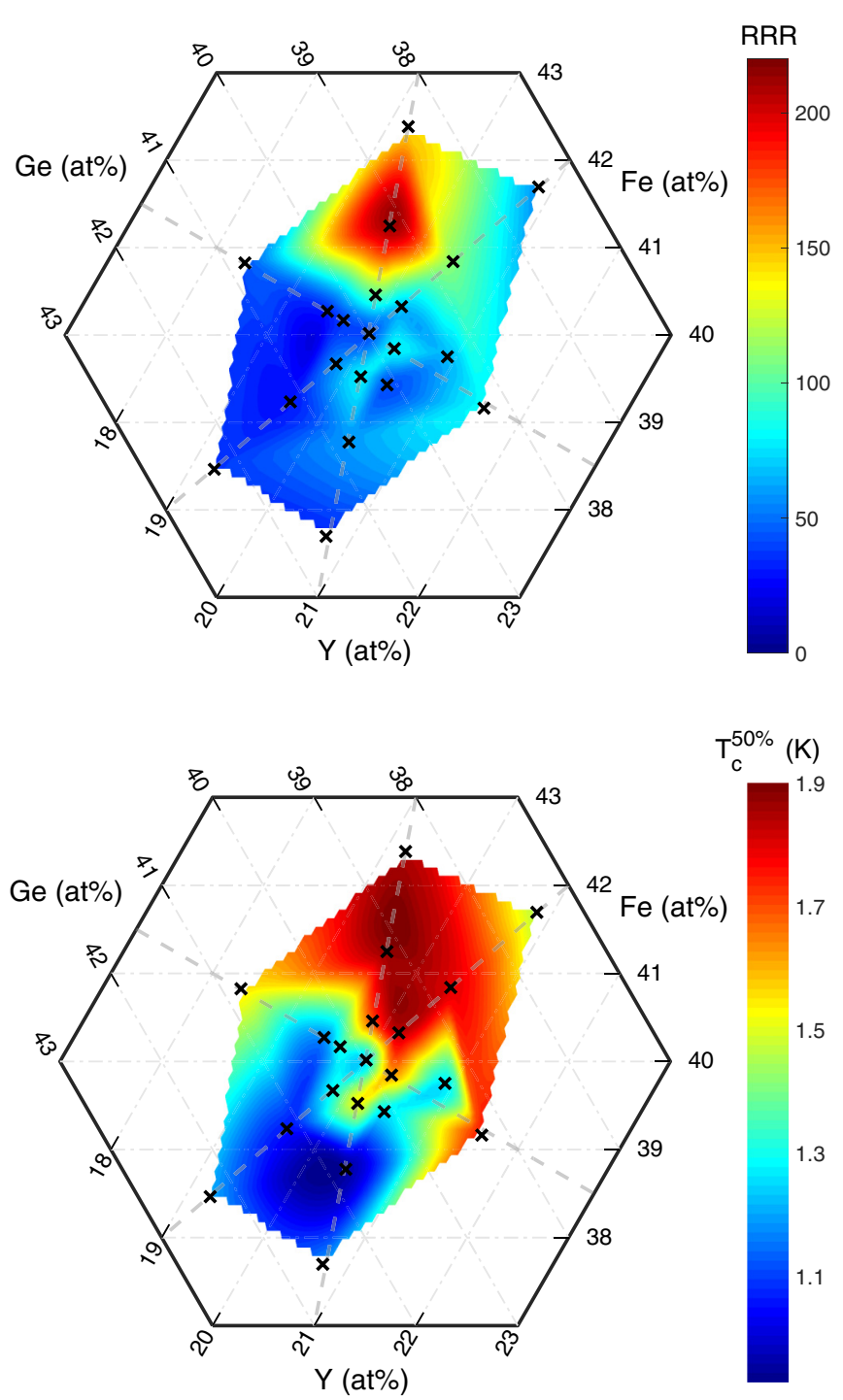

FIG. 3. Residual resistance ratio $\mathrm{RRR}=\rho(300 \mathrm{~K}) / \rho(2 \mathrm{~K})$ (upper panel) and midpoint of the resistive superconducting transition (lower panel) of annealed, polycrystalline $\mathrm{Y}_{1+x}\left(\mathrm{Fe}_{1+y} \mathrm{Ge}_{1+z}\right)_{2}$ on a ternary diagram. Data shown in the ternary plots represent the highest values of RRR or $T_{c}$ observed for nominal compositions marked by crosses, and interpolated in between (see [16] for tables of compositions and for data on both as-grown and annealed samples).

fully suppressed when $\rho_{0}$ exceeds about $1.6 \mu \Omega \mathrm{cm}$. This contrasts with the threshold of $10 \mu \Omega \mathrm{cm}$ for full resistive transitions. The resistive transition, although eventually suppressed, is therefore more robust than might be expected, which may indicate that percolating superconducting paths through high-purity regions of a sample can be found even in samples in which the averaged resistivity ratio is comparatively low. The experimental observation that residual resistivities of less than $2 \mu \Omega \mathrm{cm}$ are required to observe thermodynamic signatures of the superconducting phase transition, by contrast, is fully in line with this analysis.

Having established the central role of impurity scattering in suppressing superconductivity in $\mathrm{YFe}_{2} \mathrm{Ge}_{2}$, we now examine the influence of growth conditions on sample quality. The variation of resistance ratio RRR and resistive $T_{c}$ with nominal

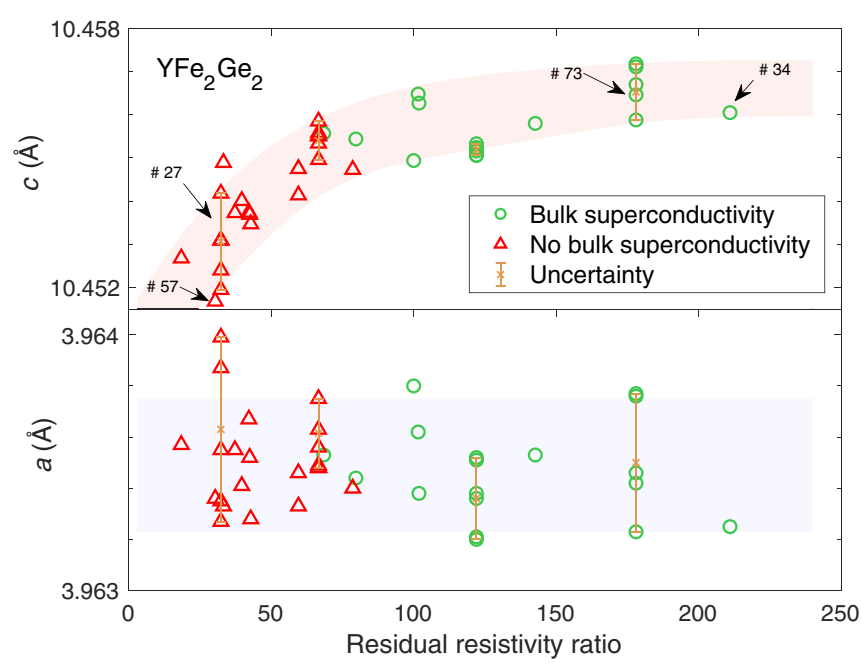

FIG. 4. Lattice constants $c$ (upper panel) and $a$ (lower panel) of the majority phase in each batch of samples as obtained by XRD refinement, versus the corresponding RRR values. Green circles (red triangles) indicate that superconducting heat capacity anomalies have (have not) been observed. Error bars are estimated from repeated measurements on the selected batches.

composition is summarized in Fig. 3, with additional detail, in particular the corresponding data for as-grown samples, available in [16]. Our data indicate that the primary influence on sample quality is the ratio of Fe vs Ge content in the melt: along the line of constant $\mathrm{Y}$ content (running diagonally to the top-right of the figure), both RRR and $T_{c}$ show the largest variation, and growth from an Fe-rich, Ge-poor melt results in higher RRRs and $T_{c}$ 's. In particular, the highest RRR of 211 was observed in a sample selected from the annealed $\mathrm{Y}\left(\mathrm{Fe}_{1.05} \mathrm{Ge}\right)_{2}$ ingot (No. 34) which also exhibits an enhanced $T_{c}$ of $1.87 \mathrm{~K}$ [8].

The nature of the $\mathrm{YFe}_{2} \mathrm{Ge}_{2}$ samples grown from melts of varying composition has been analyzed by powder XRD and EDS. Because $\mathrm{YFe}_{2} \mathrm{Ge}_{2}$ has a narrow homogeneity range, as documented by the high RRR values achieved after annealing, alien phases must be present in nominally off-stoichiometric samples, and have indeed been detected and characterized [16]. However, the observed signatures of superconductivity in $\mathrm{YFe}_{2} \mathrm{Ge}_{2}$ cannot be attributed to these alien phases, because volume superconductivity has also been detected in samples with negligible alien phase content and in samples in which the alien phases are known to be nonsuperconducting (e.g., [8]). X-ray lattice constant measurements reveal a clear correlation between the lattice parameters of samples with varying nominal composition and their corresponding maximal RRR (Fig. 4). Whereas the $a$-axis lattice parameter is the same in different ingots, a trend toward larger $c$-axis lattice parameters is observed for higher-quality samples, which also tend to show bulk superconductivity.

In order to investigate a possible link between $c$-axis lattice parameter and sample composition, we performed EDS measurements on samples showing the smallest (Nos. 27 and 57) and the largest (Nos. 34 and 73) $c$ parameters. As shown in Table I, the Fe:Ge ratios are noticeably higher in Nos. 34 and 73, both of which have higher RRR and larger 
TABLE I. Fe/Ge concentration ratios determined by EDS [16] for four $\mathrm{YFe}_{2} \mathrm{Ge}_{2}$ ingots of varying RRR, as shown in Fig. 4.

\begin{tabular}{lcccc}
\hline \hline Ingot No. & 27 & 57 & 34 & 73 \\
Fe:Ge & $0.965(2)$ & $0.967(2)$ & $0.984(2)$ & $0.985(2)$ \\
\hline \hline
\end{tabular}

c parameters than in Nos. 27 and 57. Samples with RRR as high as 200 must be close to the ideal stoichiometry. The EDS results therefore point toward a lack of Fe in samples with lower RRR, which could arise from substitution of $\mathrm{Fe}$ by $\mathrm{Ge}$ on $\mathrm{Fe}$ sites or from $\mathrm{Fe}$ vacancies. Because the metallic radius of $\mathrm{Fe}$ is slightly larger than the covalent radius of $\mathrm{Ge}$, either possibility would explain the reduction of the $c$-axis lattice parameter in the lower quality, Fe-poor samples. Based on these findings we expect that the homogeneity range of $\mathrm{YFe}_{2} \mathrm{Ge}_{2}$ is elongated along the $\mathrm{Fe} / \mathrm{Ge}$ (constant $\mathrm{Y}$ content) axis, but its Fe-rich border passes very close to the stoichiometric position, whereas its Ge-rich border is well away from stoichiometry. The highest-quality samples are then grown from an iron-rich melt.

The observation that the highest $T_{c}$ and the clearest thermodynamic signatures of superconductivity are found in the samples with the highest RRR points toward unconventional superconductivity. This and the unusually high Sommerfeld coefficient in $\mathrm{YFe}_{2} \mathrm{Ge}_{2}$ prompt the question whether this material is close to a magnetic quantum critical point (QCP) and whether magnetic order could be induced in it. Varying the $\mathrm{Fe} / \mathrm{Ge}$ ratio opens up the possibility of tuning the electronic and magnetic properties of $\mathrm{YFe}_{2} \mathrm{Ge}_{2}$, which according to DFT calculations $[3,4,25]$ and recent NMR and neutron-scattering experiments $[7,26]$ is finely balanced close to several types of magnetic order. In contrast to $\mathrm{CeCu}_{2} \mathrm{Si}_{2}$, which can be tuned between magnetically ordered and fully superconducting low temperature states by varying the sample composition [27], no magnetic transitions have been observed in any of our samples of $\mathrm{YFe}_{2} \mathrm{Ge}_{2}$. This is consistent with the comparatively high RRR observed in all annealed samples, which points toward a homogeneity range that is too narrow to include the magnetic sector of the low temperature phase diagram. A magnetic quantum critical point can be accessed by doping, as in the alloying series $(\mathrm{Lu} / \mathrm{Y}) \mathrm{Fe}_{2} \mathrm{Ge}_{2}$ [28], but no doped samples have shown any signatures of superconductivity, consistent with the view that disorder scattering rapidly suppresses superconductivity in $\mathrm{YFe}_{2} \mathrm{Ge}_{2}$.

Although there is no evidence for long-range magnetic order in any of our samples, we observe pronounced upturns in $C(T) / T$ below $2 \mathrm{~K}$ [Fig. 1(d)] in the more disordered samples $(\mathrm{RRR}<60)$. Similar upturns in $C / T$ are also evident in earlier samples of $\mathrm{YFe}_{2} \mathrm{Ge}_{2}$ which do not display bulk superconductivity $[1,2,15]$, and they are found in as-grown samples with iron-rich as well as iron-poor nominal composition, indicating that this phenomenon is not primarily a consequence of composition tuning. Because these upturns persist under applied magnetic field up to $2.5 \mathrm{~T}$, above the resistive upper critical field of $\mathrm{YFe}_{2} \mathrm{Ge}_{2}$, they are unlikely to be caused by a superconducting transition. The low- $T$ heat capacity upturns are absent in the annealed samples with the lowest disorder levels, which also show bulk superconductivity [e.g., Figs. 1(e) and 1(f)], even if in some cases upturns were observed in the more disordered, as-grown parts of ingots with the same composition. The $C(T)$ upturns therefore cannot be attributed to a clean-limit magnetic QCP but may instead be caused by magnetically ordered rare regions, or Griffiths-phase phenomena, which arise naturally in disordered and inhomogeneous samples near a clean-limit QCP [29]. Although it has not been possible to access a magnetically ordered state by shifting the $\mathrm{Fe} / \mathrm{Ge}$ ratio within the very narrow homogeneity range of $\mathrm{YFe}_{2} \mathrm{Ge}_{2}$, these findings on disordered samples are consistent with the view that this material is very close to a magnetic instability.

Our study demonstrates that the lowest disorder polycrystals of $\mathrm{YFe}_{2} \mathrm{Ge}_{2}$ can be grown by shifting the Fe/Ge ratio in the melt to favor full Fe occupancy on the Fe sites. Followed by annealing, which minimizes antisite disorder, this produces samples with residual resistivities as low as $\sim 1 \mu \Omega \mathrm{cm}$, which exhibit superconducting anomalies in the heat capacity as well as in the resistivity. The strong correlation between the residual resistivity and $T_{c}$ (Fig. 2) found by studying dozens of samples with a wide range of nominal compositions, both asgrown and annealed, is reminiscent of well-known unconventional superconductors such as $\mathrm{Sr}_{2} \mathrm{RuO}_{4}$ [20], $\mathrm{YBa}_{2} \mathrm{Cu}_{3} \mathrm{O}_{7-\delta}$ [30], and $\mathrm{CeCoIn}_{5}$ [22]. Even the purest samples display a striking separation between the resistive $T_{c}$, which is as high as $\simeq 1.87 \mathrm{~K}$, and the heat capacity anomaly, which occurs below about $1.1 \mathrm{~K}$. This separation may be attributed to spatial inhomogeneity within the sample, but the nature of this inhomogeneity - whether composition, disorder level, or strain (e.g., [31])—requires further investigation.

All data needed to evaluate the conclusions in the paper are present in the paper, the Supplementary Materials, and the Data Repository at the University of Cambridge and can be downloaded from [34].

We thank, in particular, C. Geibel, F. Steglich, G. Lonzarich, S. Dutton, and J. Baglo for helpful discussions. The work was supported by the EPSRC of the UK (Grants No. EP/K012894 and EP/P023290/1) and by Trinity College.
[1] Y. Zou, Z. Feng, P. W. Logg, J. Chen, G. Lampronti, and F. M. Grosche, Phys. Status Solidi RRL 8, 928 (2014).

[2] M. Avila, S. Bud'ko, and P. Canfield, J. Magn. Magn. Mater. 270, 51 (2004).

[3] A. Subedi, Phys. Rev. B 89, 024504 (2014).

[4] D. J. Singh, Phys. Rev. B 89, 024505 (2014).
[5] D. F. Xu, D. W. Shen, D. Zhu, J. Jiang, B. P. Xie, Q. S. Wang, B. Y. Pan, P. Dudin, T. K. Kim, M. Hoesch, J. Zhao, X. G. Wan, and D. L. Feng, Phys. Rev. B 93, 024506 (2016).

[6] N. Sirica, F. Bondino, S. Nappini, I. Píš, L. Poudel, A. D. Christianson, D. Mandrus, D. J. Singh, and N. Mannella, Phys. Rev. B 91, 121102 (2015). 
[7] H. Wo, Q. Wang, Y. Shen, X. Zhang, Y. Hao, Y. Feng, S. Shen, Z. He, B. Pan, W. Wang, K. Nakajima, S. Ohira-Kawamura, P. Steffens, M. Boehm, K. Schmalzl, T. R. Forrest, M. Matsuda, Y. Zhao, J. W. Lynn, Z. Yin, and J. Zhao, arXiv:1808.07262.

[8] J. Chen, K. Semeniuk, Z. Feng, P. Reiss, P. Brown, Y. Zou, P. W. Logg, G. I. Lampronti, and F. M. Grosche, Phys. Rev. Lett. 116, 127001 (2016).

[9] D. Guterding, H. O. Jeschke, I. I. Mazin, J. K. Glasbrenner, E. Bascones, and R. Valentí, Phys. Rev. Lett. 118, 017204 (2017).

[10] I. Felner, B. Lv, K. Zhao, and C. W. Chu, J. Supercond. Novel Magn. 28, 1207 (2015).

[11] A. P. Pikul, M. Samsel-Czekała, G. Chajewski, T. Romanova, A. Hackemer, R. Gorzelniak, P. Wiśniewski, and D. Kaczorowski, J. Phys.: Condens. Matter 29, 195602 (2017).

[12] G. Chajewski, M. Samsel-Czekała, A. Hackemer, P. Wiśniewski, A. P. Pikul, and D. Kaczorowski, Physica B: Condens. Matter 536, 767 (2018).

[13] M. Samsel-Czekała, G. Chajewski, P. Wiśniewski, T. Romanova, A. Hackemer, R. Gorzelniak, A. P. Pikul, and D. Kaczorowski, Physica B: Condens. Matter 536, 816 (2018).

[14] F. Bernardini, G. Garbarino, A. Sulpice, M. Núñez-Regueiro, E. Gaudin, B. Chevalier, M. A. Méasson, A. Cano, and S. Tence, Phys. Rev. B 97, 100504(R) (2018).

[15] H. Kim, S. Ran, E. D. Mun, H. Hodovanets, M. A. Tanatar, R. Prozorov, S. L. Bud'ko, and P. C. Canfield, Philos. Mag. 95, 804 (2015).

[16] See Supplemental Material at http://link.aps.org/supplemental/ 10.1103/PhysRevB.99.020501, which includes Refs. [8,32,33], for further details of the nominal composition ternary phase diagram, of electrical resistivity data from as-grown and annealed samples, of the calculation of the quasiparticle scattering rate and the mean free path, and of energy dispersive spectroscopy measurements .

[17] A. A. Abrikosov and L. P. Gorkov, J. Exptl. Theoret. Phys. 39, 1781 (1960) [Sov. Phys. JETP 12, 1243 (1961)].

[18] R. J. Radtke, K. Levin, H. B. Schüttler, and M. R. Norman, Phys. Rev. B 48, 653(R) (1993).
[19] H. A. Blackstead and J. D. Dow, Phys. Lett. A 206, 107 (1995).

[20] A. P. Mackenzie, R. K. W. Haselwimmer, A. W. Tyler, G. G. Lonzarich, Y. Mori, S. Nishizaki, and Y. Maeno, Phys. Rev. Lett. 80, 161 (1998).

[21] Z. Q. Mao, Y. Mori, and Y. Maeno, Phys. Rev. B 60, 610 (1999).

[22] E. D. Bauer, F. Ronning, C. Capan, M. J. Graf, D. Vandervelde, H. Q. Yuan, M. B. Salamon, D. J. Mixson, N. O. Moreno, S. R. Brown, J. D. Thompson, R. Movshovich, M. F. Hundley, J. L. Sarrao, P. G. Pagliuso, and S. M. Kauzlarich, Phys. Rev. B 73, 245109 (2006).

[23] Y. Kitaoka, K. Ishida, and K. Asayama, J. Phys. Soc. Jpn. 63, 2052 (1994).

[24] A. J. Millis, S. Sachdev, and C. M. Varma, Phys. Rev. B 37, 4975 (1988).

[25] G. Wang and X. Shi, Comput. Mater. Sci. 121, 48 (2016).

[26] J. Srpčič, P. Jeglič, I. Felner, B. Lv, C. W. Chu, and D. Arčon, Phys. Rev. B 96, 174430 (2017).

[27] P. Gegenwart, C. Langhammer, C. Geibel, R. Helfrich, M. Lang, G. Sparn, F. Steglich, R. Horn, L. Donnevert, A. Link, and W. Assmus, Phys. Rev. Lett. 81, 1501 (1998).

[28] S. Ran, S. L. Bud'ko, and P. C. Canfield, Philos. Mag. 91, 4388 (2011).

[29] T. Vojta, J. Phys. A: Math. Gen. 39, R143 (2006).

[30] F. Rullier-Albenque, H. Alloul, and R. Tourbot, Phys. Rev. Lett. 91, 047001 (2003).

[31] M. D. Bachmann, G. M. Ferguson, F. Theuss, T. Meng, C. Putzke, T. Helm, K. R. Shirer, Y.-S. Li, K. A. Modic, M. Nicklas, M. Koenig, D. Low, S. Ghosh, A. P. Mackenzie, F. Arnold, E. Hassinger, R. D. Mcdonald, L. E. Winter, E. D. Bauer, F. Ronning, B. J. Ramshaw, K. C. Nowack, and P. J. W. Moll, arXiv:1807.05079.

[32] P. Schobinger-Papamantellos, J. Rodriguez-Carvajal, G. Andre, N. P. Duong, K. H. J. Buschow, and P. Tolédano, J. Magn. Magn. Mater. 236, 14 (2001).

[33] B. Chakraborty, W. E. Pickett, and P. B. Allen, Phys. Rev. B 14, 3227 (1976).

[34] https://doi.org/10.17863/CAM.33904. 